\title{
Focusing X-Rays with Curved Multiplate Crystal Cavity
}

\author{
Ying-Yi Chang, ${ }^{1}$ Sung-Yu Chen, ${ }^{1}$ Shih-Chang Weng, ${ }^{1}$ Chia-Hung Chu, ${ }^{1}$ Mau-Tsu Tang, ${ }^{2}$ \\ Yuriy Stetsko, ${ }^{2}$ Bo-Yuan Shew, ${ }^{2}$ Makina Yabashi, ${ }^{3}$ and Shih-Lin Chang ${ }^{1}$ \\ ${ }^{1}$ Department of Physics, National Tsing Hua University, Hsinchu 300, Taiwan \\ ${ }^{2}$ Experiment Facility Division, National Synchrotron Radiation Research Center, Hsinchu 300, Taiwan \\ ${ }^{3}$ RIKEN, XFEL Project Head Office, Experimental Facility Group, Spring-8/RIKEN, Mikazuki, Hyogo 679-5148, Japan
}

Correspondence should be addressed to Shih-Lin Chang, slchang@phys.nthu.edu.tw

Received 26 January 2010; Accepted 23 July 2010

Academic Editor: Gene Ice

Copyright () 2010 Ying-Yi Chang et al. This is an open access article distributed under the Creative Commons Attribution License, which permits unrestricted use, distribution, and reproduction in any medium, provided the original work is properly cited.

\begin{abstract}
An overview is given of the study on X-ray focusing using the Fabry-Perot type multi-plate silicon crystal cavities consisting of compound refractive lenses. Silicon (12 40 ) is used as the back reflection for cavity resonance at the photon energy of $14.4388 \mathrm{keV}$. Measurements of focal length of the transmitted beam through the crystal cavities show enhanced focusing effect due to the presence of back diffraction. Also, an incident beam with ultrahigh energy resolution can improve the focusing owing to the wider acceptance angle of the back diffraction. Considerations based on the excitation of dispersion surface within the framework of $\mathrm{X}$-ray dynamical diffraction theory are also presented to reveal the origin of this enhanced focusing.
\end{abstract}

\section{Introduction}

Focusing X-rays is usually considered very difficult because the refractive index of $\mathrm{X}$-rays is smaller than and very close to unity. The advances in technology development have led to X-ray focusing using polycapillaries [1] and zone plates [2]. In 1996, Snigirev et al. succeeded in focusing high-energy $\mathrm{X}$-rays by the so-called compound refractive lenses (CRL) of spherical shape [3]. Later, the CRL of parabolic shape have been developed to eliminate the spherical aberration and other distortion and Kinoform lenses have also been used to diminish the absorption [4]. Moreover, several kinds of CRL were proposed to exceed the critical angle limit $[5,6]$. Very recently, two- and multi-plate X-ray cavities of silicon have been realized using X-ray back reflection and X-ray interference fringes due to cavity resonance been observed [7, 8]. In addition, attempts to combine this Fabry-Perot type crystal cavity with CRL lenses of circular [9] and parabolic shapes [10] for X-ray focusing have been pursued. In this paper, an overview is given of this development of utilizing crystal cavities for focusing X-rays. Also, the difference in beam-focusing between the use of back diffraction and the conventional optical refraction will be addressed.
In the following, we will first briefly describe the cavity resonance in Fabry-Perot type crystal cavity via back diffraction from perfect silicon crystals.

\section{X-Ray Fabry-Perot Resonator and Back Diffaction}

Hard X-ray Fabry-Perot resonator is composed of two or multiple crystal plates of a monolithic Si crystal. An incident $\mathrm{X}$-ray beam is reflected back and forth within the gap between the two adjacent crystal plates via a back reflection, whose Bragg angle is 90 degrees $[7,8,11,12]$. The interaction among the forward transmitted and back reflected beams gives rise to interference fringes due to cavity resonance, provided that the required coherent condition is satisfied. That is, the longitudinal coherent length $l_{L}$ is greater than two times the effective gap distance $d_{e}$, that is, $l_{L}>2 d_{e}$, where $d_{e}=d+t, d$ is the thickness of the crystal plate and $t$ the gap distance $[7,8]$. Experimentally, interference fringes in angle and energy scans are the convincing proof for the cavity resonance effects. The distance between two adjacent maxima of the transmitted beams in an energy scan is the so-called free spectral range $\left(E_{d}\right)$, defined as $E_{d}=h c / 2 d_{e}$ 


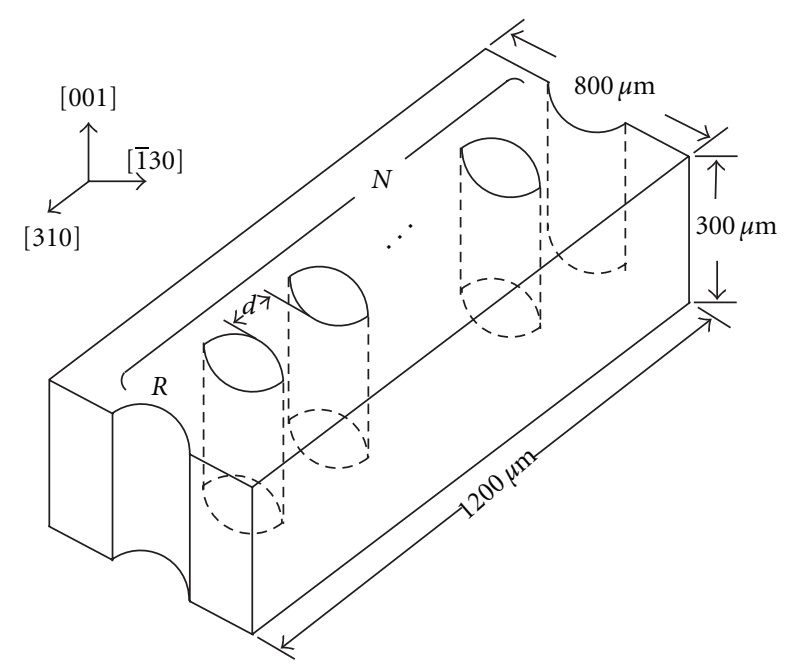

FIGURE 1: Schematic of focusing crystal cavity with compound parabolic lenses: Each hole also serves as a crystal cavity of the Fabry-Perot type.

TABLE 1: The parameters of the focusing crystal cavity devices.

\begin{tabular}{lcccc}
\hline Device & $d(\mu \mathrm{m})$ & $R(\mu \mathrm{m})$ & $N$ & $f_{\text {optics }}(\mathrm{mm})$ \\
\hline No. 1 & 10 & 40 & 12 & 715 \\
No. 2 & 10 & 50 & 13 & 800 \\
\hline
\end{tabular}

where $h$ is the Planck constant. This distance can be verified experimentally.

If the surface of the crystal plates of a cavity (resonator) is changed from flat to concaved surface, then the cavity could act as an X-ray lens, similar to the compound refractive lens. With this idea, the following focusing cavities and related $\mathrm{X}$ ray diffraction experiments are designed.

\section{Sample Preparation and Experimental Setup}

Figure 1 is the schematic of a multi-plate crystal cavity for Xray focusing. The shape of curved multiple plates is similar to the conventional parabolic refractive X-ray lenses. The crystal devices were manufactured from [001] silicon wafers using microelectronic lithography processes and dry etched with reactive ions. Several holes with parabolic cross-section were made on the wafer such that a serious of concave lenses are lined up along the [310] direction. The (12 40$)$ was used as the back reflection for $14.4388 \mathrm{keV} \mathrm{X}$-rays. The structures were $300 \mu \mathrm{m}$ deep along the [001] direction. There were two different designs for the crystal devices, No.1 and No.2. The design parameters are listed in Table 1 , where $R$ is the radius of the parabola apex, $d$ the distance between the two adjacent holes, and $N$ the number of the lenses. The lens formula $f_{\text {optics }}=R / 2 N \delta$ was used for the design, where $f_{\text {optics }}$ is focal length and the corresponding refractive index is $n=1-\delta-$ $i \beta$. For $14.4388 \mathrm{keV}$ photon energy, the real and imaginary corrections of the refractive index are $\delta=2.33 \times 10^{-6}$ and $\beta=1.72 \times 10^{-8}$. The ideal focal distances are 715 and $820 \mathrm{~mm}$ for No. 1 and No. 2 cavities, respectively.

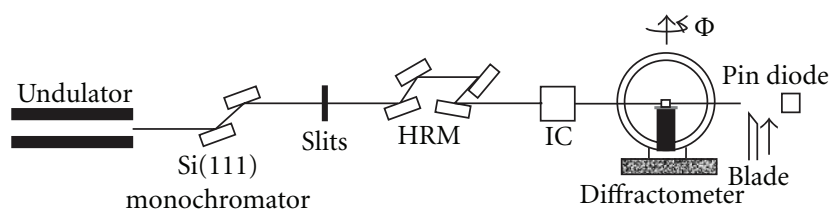

Figure 2: Experimental setup: The incident radiation was monochromatized by a $\operatorname{Si}(111)$ double-crystal first and then by a highresolution monochromator (HRM) at $14.4388 \mathrm{keV}$. The focusing crystal cavity is placed at the center of an 8-circle diffractometer. An ion chamber (IC) and a pin diode were used to monitor the back-diffracted (1240) and the forward-transmitted (000) beam.

Focal length measurements were carried out on the Taiwan undulator beamline BL12XU at the Spring-8 synchrotron facility in Japan. Figure 2 is the experimental layout. The storage ring was operating at $8 \mathrm{GeV}$ and $100 \mathrm{~mA}$. The synchrotron radiation was first monochromatized to the X-ray energy close to $14.4388 \mathrm{keV}$ using a $\mathrm{Si}(111)$ double-crystal monochromator. The X-rays were then further tuned to have the energy resolution $\Delta E / E \sim 2.5 \times$ $10^{-8}$ at $14.4388 \mathrm{keV}$ with a four-crystal high-resolution monochromator (HRM). The HRM was composed of two pairs of asymmetric (422) and (11 553 ) reflection planes of Si [13]. Experimentally, focal lengths were measured with and without the HRM to investigate the energyresolution dependence of focusing. The crystal device was mounted on a goniometric head located at the center of a Huber 8-circle diffractometer. The incident beam was in the $\left[\begin{array}{ll}-3-1 & 0\end{array}\right]$ direction normal to the device. The forwardtransmitted and the back-reflected beams were monitored by an ion chamber and a pin-diode, respectively. A knifeedge of $500 \AA$ surface roughness was used to measure the beam size of the forward-transmitted beam through the crystal cavity at several positions along the transmitted beam direction.

\section{Results}

The energy resolution of the incident beam without using the HRM was about $\Delta E / E \sim 1.4 \times 10^{-4}$ and $\Delta E \sim 2 \mathrm{eV}$ at $14.4388 \mathrm{keV}$. Under this condition, both the horizontal tilt, $\Delta \varphi$, around [001] and the vertical rotation, $\Delta \theta$, around $\left[\begin{array}{lll}-1 & 3 & 0\end{array}\right]$ of the device No. 1 gave the intensity distribution of the transmitted beam as a dip as shown in Figures 3(a) and 3(b). The high background plateau was the intensity of the (000) beam without diffraction. This means that during the back diffraction of (1240) at the position, $\Delta \theta=0^{\circ}$ and $\Delta \varphi=0^{\circ}$, the intensity of the (000) beam was taken away by the (12 40 ) reflection. However, due to the insufficient energy resolution, we were sure that the diffraction condition of the (12 40 ) back reflection was not exactly fulfilled because the dip was too sharp. As will be clear, the correct profile of the (12 40 ) back reflection is a much wider dip (see Figure 4(a)). Nevertheless, it is yet worth investigating the beam-focusing effect under general diffraction conditions with modest energy resolution. 


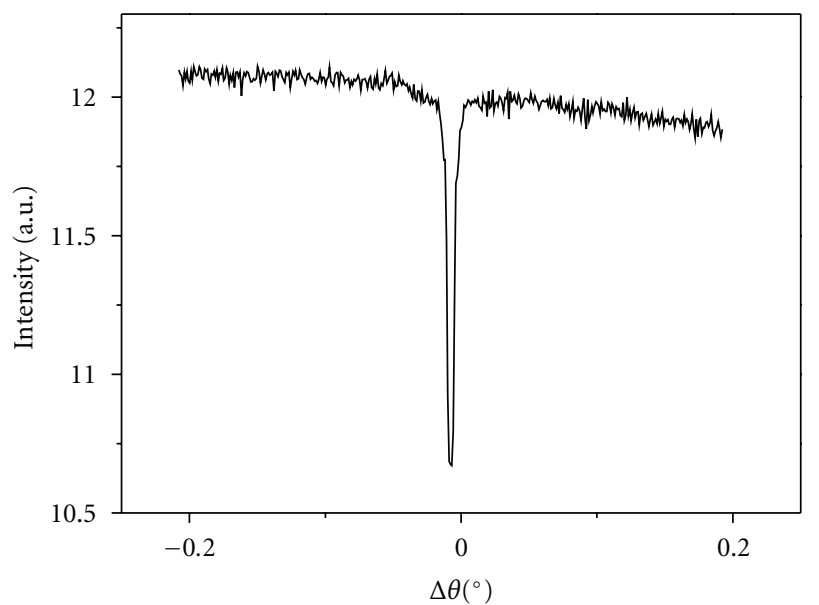

(a)

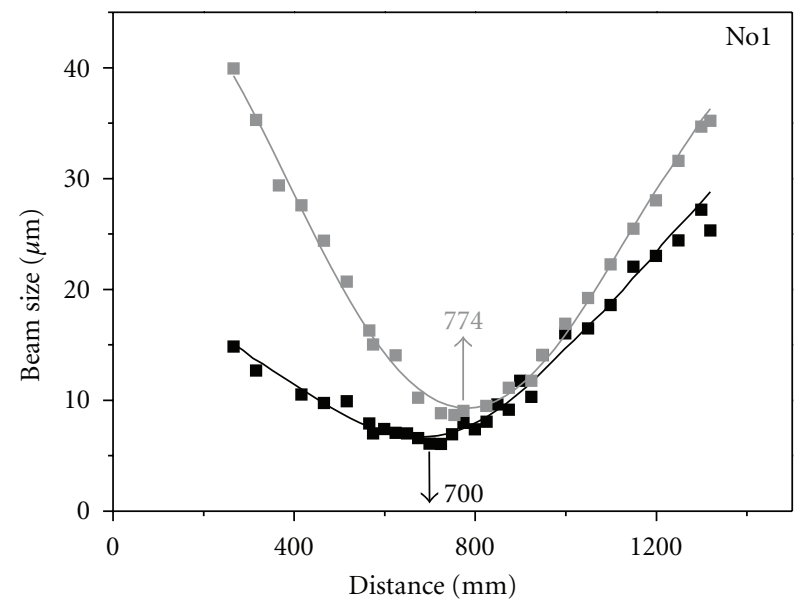

(c)

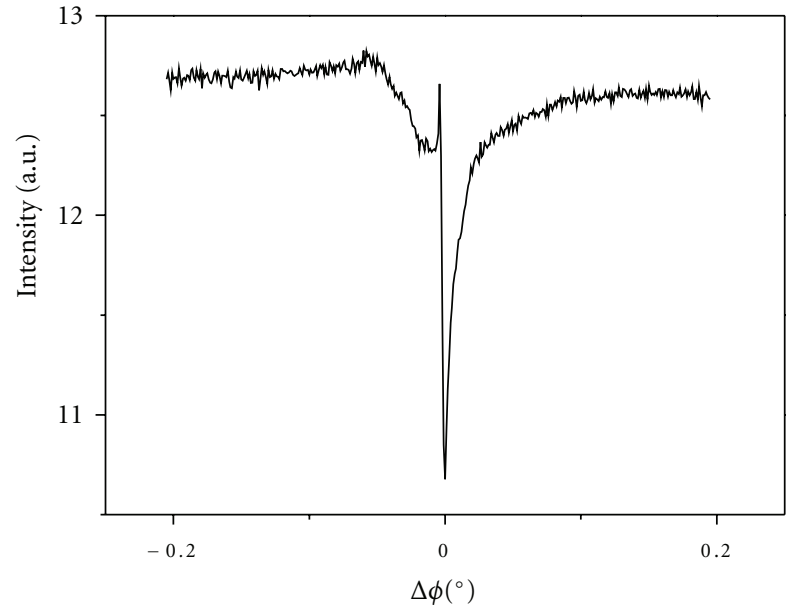

(b)

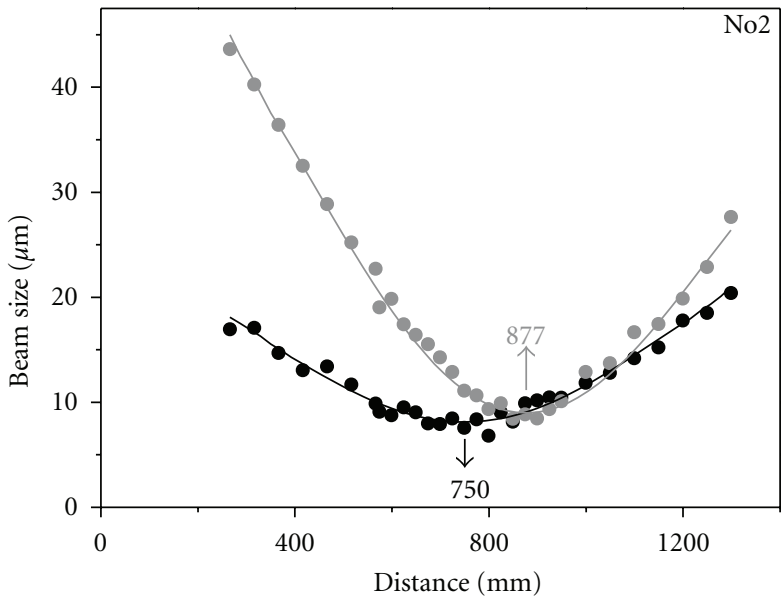

(d)

Figure 3: (a) The $\Delta \theta$ scan at $\Delta \varphi=0$ and (b) the $\Delta \varphi$ scan at $\Delta \theta=0$ of the transmitted beam through the crystal device No. 1. (c) and (d) The beam sizes of the transmitted beams through the crystal devices, No. 1 and No. 2, with (black dots) and without the back diffraction (gray dots) versus the distance from the exit end of the crystal devices.

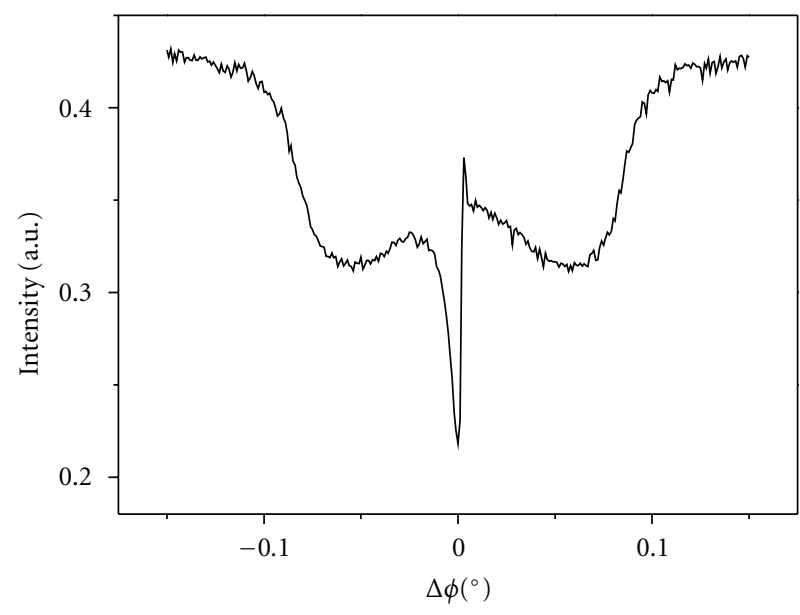

(a)

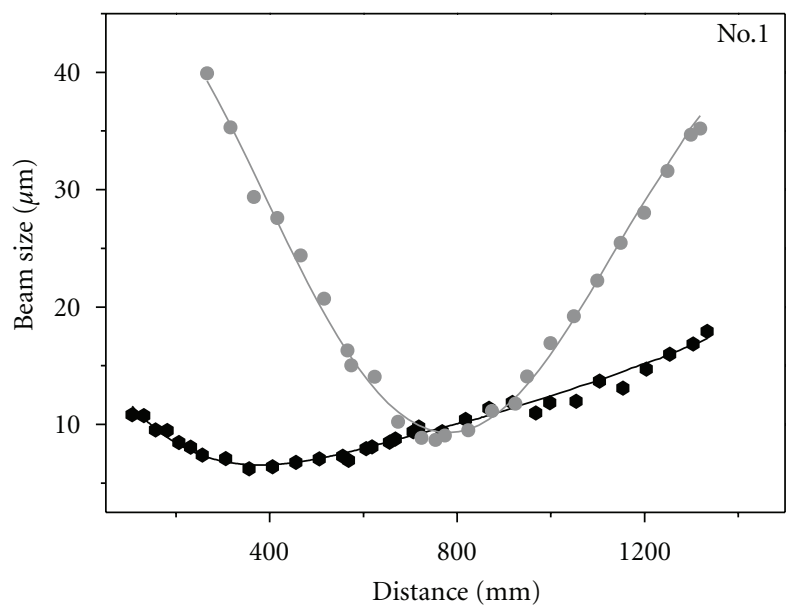

(b)

Figure 4: (a) The $\Delta \varphi$ scan at $\Delta \theta=0$ of the (12 4 0) back diffraction for the crystal device No. 1. (b) The beam sizes of the transmitted beam through the crystal device No. 1 with (black dots) and without back diffraction (gray dots) versus the distance from the exit end of the crystal device. 
Figures $3(\mathrm{c})$ and $3(\mathrm{~d})$ are the measured sizes of the transmitted beam at various positions versus the distance of the measured position from the exit end of the crystal devices, No. 1 and No. 2, respectively. The gray dots and black dots show respectively the beam sizes measured at the angular position, $\Delta \theta=0^{\circ}$ and $\Delta \varphi=-0.15^{\circ}$, at which no diffraction takes place and at the position, $\Delta \theta=0^{\circ}$ and $\Delta \varphi=0^{\circ}$, at which the (1240) diffraction occurs. When there is no diffraction, the experimental focal lengths were around 774 and $877 \mathrm{~mm}$ at the position, $\Delta \theta=0^{\circ}$ and $\Delta \varphi=-0.15^{\circ}$, for the crystal devices, No. 1 and No. 2, respectively. The results are close to the theoretical values, 715 and $820 \mathrm{~mm}$. The difference probably originates from the inaccuracy in cavity manufacturing. On the other hands, when there is a diffraction, the black dotted curves give the focal distances, 700 and $750 \mathrm{~mm}$, for the crystal devices, No. 1 and No. 2, respectively. The reduction in focal length is about $2 \sim 9 \%$. This is probably because the width of the diffraction peak, about $0.01^{\circ}$, is too narrow to maintain the back diffraction. In other words, when the parallel incident beam passes through several crystal plates, the outer part of the beam is bent more than $0.01^{\circ}$. The back diffraction condition is then no longer satisfied so that this part of the beam is bent only due to optical refraction. That is why the reduction of the focal length is very small.

After inserting the HRM into the experimental setup, the energy resolution of the incident beam was about $0.36 \mathrm{meV}$. In Figure $4(\mathrm{a})$, the horizontal tilt $(\Delta \varphi)$ of the crystal device at $\Delta \theta=0^{\circ}$ shows the intensity profile with a wide hollow of the width about $0.2^{\circ}$ against the (000) background. This indicates the occurrence of the (12 40 ) back diffraction at the photon energy almost exactly equal to $14.4388 \mathrm{keV}$. In addition, the sharp dip located right in the middle of the hollow at $\Delta \varphi=0$ results from the simultaneous presence of other 22 reflections owing to the high structural symmetry Si $[7,8]$. This 24-beam multiple diffraction also took away the transmitted and reflected intensities from the $\left(\begin{array}{lll}12 & 4 & 0\end{array}\right)$ reflection to the 22 reflected directions. Consequently the reflectivity and transmissivity of the involved X-ray beams drop considerably.

The measured beam sizes versus the distance from the end of the exit surface are shown in Figure 4(b), where the black curve was obtained at $\Delta \varphi=0^{\circ}$ when the $(12$ $40)$ back reflection and the additional 22 diffractions (24beam diffraction) were in operation $[7,8]$. And the gray curve is the measurement taken without the back reflection, namely, under a pure refraction circumstance like in the CRL situation. As can be seen, the transmitted beam was focused to the position $356 \mathrm{~mm}$ from the end of the device for the 24beam diffraction at $\Delta \varphi=0^{\circ}$, while the optical CRL focusing gave the focal length of about $774 \mathrm{~mm}$. The beam sizes at the focal points were respectively 6.2 and $9 \mu \mathrm{m}$. In other words, the focal length of the CRL was reduced by nearly a factor of 2 due the 24-beam diffraction. Moreover, under the 24beam diffraction condition, the crystal devices also preserve the characteristics of X-ray Fabry-Perot resonators. Indeed, the interference fringes were observed clearly from the device No. 1 in the photon-energy scan of the transmitted-beam (Figure 5). Since the effective gap distance is $200 \mu \mathrm{m}$ for this

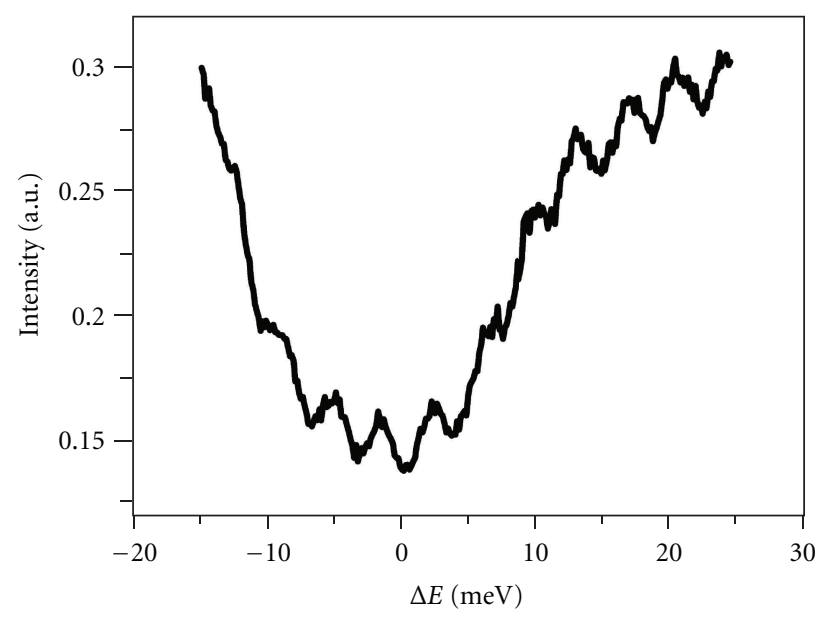

Figure 5: The energy $\Delta E$ scan at $E=14.4388 \mathrm{keV}$ of the transmitted beam through the crystal device No. 1 . The vertical axis is the normalized transmitted intensity.

crystal device, the fringe spacing, also called free spectral range $\left(E_{d}\right)$, is about $3.4 \mathrm{meV}$, which agrees with the calculated value $3.1 \mathrm{meV}$.

It is estimated that for crystal No. 1 the real gain defined in [3] is about 7.6 for refraction and 11 for back diffraction. We have also measured the intensity of the focused transmitted beam by back-diffraction which is about $18 \%$ of that of the incident beam, which is comparable with that of conventional CRL.

\section{Theoretical Analysis}

The beam-focusing effect can be understood according to the dynamical diffraction theory of X-ray back diffraction [11, 12, 14-18].

The dynamical theory of X-ray diffraction $[11,12,14-$ 16] describes the interaction of $X$-ray waves with a crystalline material, thus forming the so-called wavefields in crystal lattice. The wavefields during the X-ray diffraction can be described by Maxwell's equations. The wavefields in the crystal are assumed to take the form of Bloch waves, which lead to the fundamental equation of wavefield [14]. The secular equation of this fundamental equation gives the dispersion relation and the dispersion surface can be constructed accordingly. Dispersion surface simply sketches the relationship of the amplitudes and wavevectors of the wavefields as a function of the angular position of the crystal in the reciprocal space. As an example, Figure 6(a) is the schematic of the dispersion surface (green color) of a twobeam Bragg reflection in a wide-angle incidence geometry, where $O$ and $G$ are the reciprocal lattice points representing, respectively, the (000) and (hkl) reflection. The spheres in black are the Ewald spheres outside the crystal centered at points $O$ and $G$. After the X-rays enter the crystal, the Ewald sphere becomes smaller due to the fact that the index of refraction $n$ is smaller that unity. The intersections of the two Ewald spheres (green curves) centered at $O$ and $G$ inside the crystal are modified approximately as hyperbola 


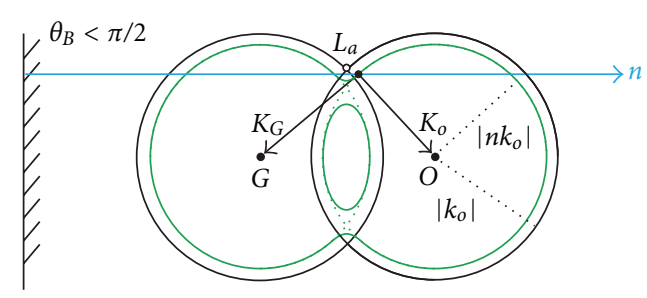

(a)

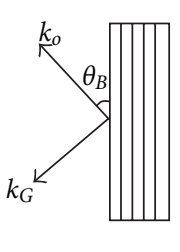

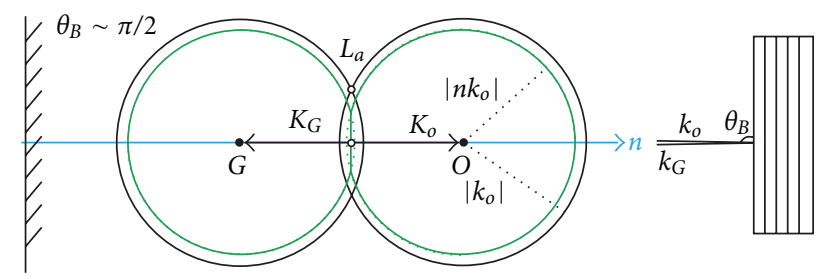

(b)

FIGURE 6: Dispersion surface projected onto the plane of incidence of a Bragg diffraction: (a) the general wide-angle incidence case with $\theta_{B}<\pi / 2$ and (b) the back diffraction case with $\theta_{B} \sim \pi / 2$. The green curves represent the dispersion surface inside the crystal. $(\hat{n}$ : the crystal surface normal; $\theta_{B}$ : the Bragg angle; $L_{a}$ : the Laue point [14]).

(green solid curves) when the Bragg's law, $\vec{K}_{G}=\vec{K}_{O}+\vec{d}_{G}$ is satisfied, where $\vec{K}_{O}$ and $\vec{K}_{G}$ are the wavevectors of the (000) and (hkl) diffracted beams inside the crystal and $\overrightarrow{d_{G}}=$ $\overrightarrow{O G}$. The corresponding wavevestors outside the crystal are $\vec{k}_{O}$ and $\vec{k}_{G}$. The dispersion surface is excited by an incident beam $\vec{k}_{O}$, whose wavevector starts from a given entrance point on the black Ewald sphere and ends at point $O$. This excitation generates the diffracted beams, $\vec{K}_{O}$ and $\vec{K}_{G}$, which have a common starting point, called tie point. The tie point and the entrance point lie along the surface normal direction $\hat{n}$ such that the continuity of the tangential components of the $\vec{K}_{O}$ and $\vec{k}_{O}$ at the crystal boundary is fulfilled. The same is true for $\vec{K}_{G}$ and $\vec{k}_{G}$. Hence, by taking the sample surface normal into account, the wavevectors inside the crystal can be determined.

However, when the Bragg angle is almost equal to $\pi / 2$, the dispersion surface has the unusual shape represented schematically in Figure 6(b). The two Ewald spheres in green color centered at points $O$ and $G$ inside the crystal are just touching and tangent to each other at the only contact point, which is also the tie point for the exact back reflection. Therefore, $\vec{K}_{O}$ and $\vec{K}_{G}$ are parallel to $\hat{n}$ and opposite to each other. Since there is only one contact region, this corresponds to only one angular range of total reflection whose angular width is $w_{D, \theta_{B} \sim \pi / 2} \sim 2 \sqrt{\left|\chi_{G}\right|}=0.0745^{\circ}$, derived from the dynamical theory [14]. It is wider than that of the general case of wide-angle incidence, $w_{D, \theta_{B}<\pi / 2} \sim\left|\chi_{G}\right|=$ $0.0014^{\circ}$, because the value of the electric susceptibility $\left|\chi_{G}\right|$ is generally about $10^{-5}$ to $10^{-7}$. Hence the acceptance angle of a back diffraction is about one order of magnitude greater than that of general cases $[11,12,14]$. This feature is useful for beam-focusing under back diffraction conditions.

We now consider the focusing effects associated with the back diffraction in the multi-plate focusing cavity based on the determination of wavevectors inside the crystal via the excitation of the dispersion surface. We will first concentrate on the back diffraction of the first crystal lens, and then extend it to the $N$ lenses. In real space (Figure 7(a)), a parallel beam of the wavevector $\vec{k}_{o 0}$ is incident on the first crystal. For simplicity, the upper part of the incident beam hits the first concave surface and generates an transmitted beam with the wavevector $\vec{K}_{O 1}$ which propagates inside the first crystal. After passing through the second (exit) surface, this $\vec{K}_{O 1}$ beam becomes the forward transmitted wavevector, $\vec{k}_{o 1}$ and exits from the first crystal. The vector $\mathbf{n}_{\mathrm{i} 1}$ and $\mathbf{n}_{\mathrm{i} 2}$ stand for the entrance and exit surface normals of the $i$ th crystal. Similar situation is encountered for the lower part of the incident beam. Although the scheme (Figure 7(a)) looks similar to optical refraction, the focusing mechanism is different due the presence of back diffraction.

The distinction between diffractive and optical focusing can be understood from the excitation of the dispersion surface in the reciprocal space. In Figure 7(b) the reciprocal lattice points $O$ and $G$ represent the incident (000) and the (12 40 0) back reflection. Because the Bragg angle $\theta_{B}$ is nearly equal to $\pi / 2$, the wavefront, $\Sigma_{O}$, of the incident beam $\vec{k}_{o 0}\left(=\overrightarrow{E_{0} O}\right)$ is almost perpendicular to the reciprocal lattice vector $\overrightarrow{O G}$. $\mathrm{E}_{0}$ is the entrance point of the beam $\vec{k}_{o 0}$. Considering the continuity of the tangential components of the wavevectors inside and outside the crystal at the boundary, the incident beam $\vec{k}_{o 0}$ excites the dispersion surface at the tie point $T_{1}$ along the first surface normal $\mathbf{n}_{11}$, thus generating a forward transmitted wave, $\overrightarrow{T_{1} O}(=$ $\vec{K}_{O 1}$ )inside the first crystal. When the beam $\vec{K}_{O 1}$ arrives at the first exit surface, it generates the outgoing wavevector, $\overrightarrow{E_{1} O}\left(=\vec{k}_{o 1}\right)$ along the exit surface normal $\mathbf{n}_{12}$. In contrast to the back diffraction, the grey dashed line represents the dispersion surface for optical refraction, projected onto the plane of the incidence of the (12 40$)$ reflection. In this case, the incident beam $\vec{k}_{o 0}$ excites the (grey) dispersion surface and generates a refracted beam $\overrightarrow{g_{1} O}$ inside the first crystal. Subsequently, an outgoing exit beam $\overrightarrow{r_{1} O}$ is produced. By comparing the degree of inclination of the wavevectors involved in diffraction and refraction, it is clear that the diffracted beams $\overrightarrow{T_{1} O}$ hand $\overrightarrow{E_{1} O}$ are more focused onto point $O$ than the refracted beams $\overrightarrow{g_{1} O}$ and $\overrightarrow{r_{1} O}$. Figure 7 (c) shows the focusing effects due to diffraction and refraction in ray tracing in real space for one crystal lens. Similar situation occurs for the incident beam entering in the lower half of the crystal. Namely, the beam-bending ability of back diffraction is better than that of optical refraction. Moreover, there are more dispersion sheets (96 in total) involved in the 24-beam diffraction [18]. That implies that there are more chances for the transmitted beam to focus on to point $O[10]$. 


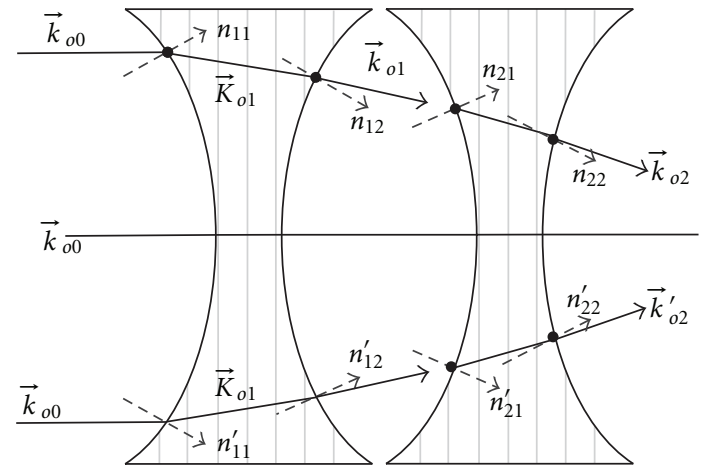

(a)

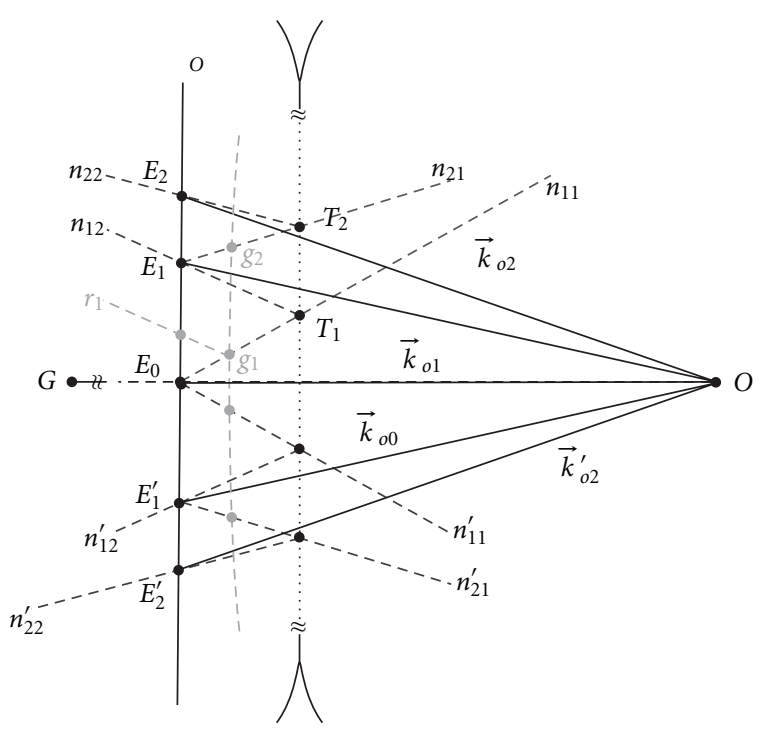

(b)

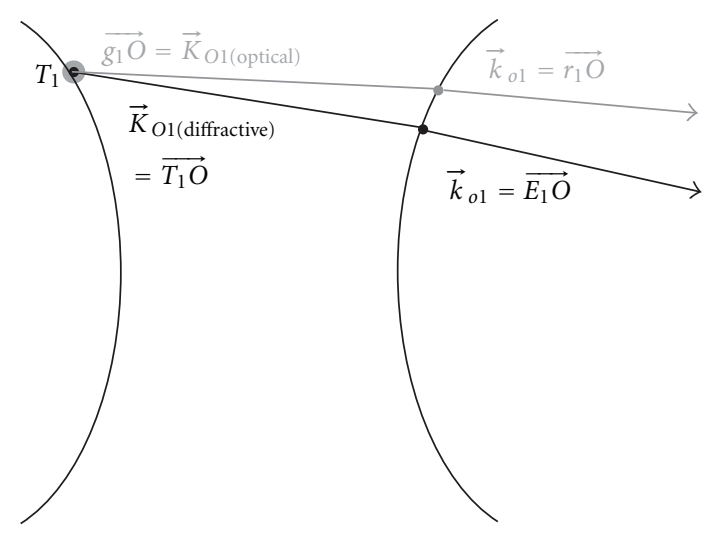

(c)

Figure 7: (a) Ray-tracing of a parallel incident beam in the first two crystal lenses in real space: the symbols with "prime" are for the lower part of the beam; (b) Schematic of the excitation of the dispersion surface projected onto the plane of incidence in reciprocal space for (a); (c) The ray-tracing of the diffracted beam (black) and the optical refracted beam (gray) for the first crystal according to the corresponding wavevectors.
Hence the transmitted beam will be focused further. This theoretical consideration can be continually applied to next crystal lens and up to the $N$ th crystal and the focusing effect due to back diffraction is expected to be much enhanced.

In summary, the dynamical effect on focusing involving back diffraction is mainly due to the excitation of the dispersion surface for curved crystal surfaces. This curved crystal boundaries make beam focusing happen. That is, this focusing effect, governed by the curvature of the curved CRL, is due to the change of the direction of the wavevector of the transmitted beam during the excitation of the dispersion surface of the diffractions involved.

\section{Conclusion}

In conclusion, we have shown $\mathrm{X}$-ray focusing using curved crystal cavity with incident X-rays of modest and ultrahigh energy-resolution. If only a conventional double crystal monochromator is employed as the beam conditioner, the focusing is less effective than the case with an ultrahigh resolution monochromator, because the latter could easily fulfill cavity resonance conditions and provide a wider acceptance angle for the incident beam. On the other hand, this type of focusing depends on the presence of diffraction. It seems that in this particular case reported here optical refraction, the two-beam back reflection, and the 24-beam diffraction give the longest, shorter, and the shortest focal length, respectively. The reduction in focal length could be $50 \%$ compared to the refraction case. Also, according to [3], the real gain of a CRL lens depends on the absorption, real focus size, effective lens aperture, image distance, and source distance. Since the focus size for back diffraction is smaller than for refraction, the gain for back diffraction is larger than that for refraction. This distinct feature could be useful for high resolution diffraction, scattering, and imaging investigations. However, because back diffraction takes place at a specific energy and the required energy resolution is very strict, the use of this type of crystal devices as a focus tool might be limited to some specific photon energies for a given crystal used for making the devices.

\section{Acknowledgements}

The authors thank C.-C. Chen, N. Hiraoka of NSRRC for technical supports, and the Ministry of Education and National Science Council (NSC) of Taiwan, for financial supports. Special funding from the NSC Academic Summit Program is also gratefully acknowledged.

\section{References}

[1] C. A. MacDonald and W. M. Gibson, "Applications and advances in polycapillary optics," X-Ray Spectrometry, vol. 32, no. 3, pp. 258-268, 2003.

[2] G.-C. Yin, Y.-F. Song, M.-T. Tang et al., " $30 \mathrm{~nm}$ resolution $\mathrm{X}$ ray imaging at $8 \mathrm{keV}$ using third order diffraction of a zone plate lens objective in a transmission microscope," Applied Physics Letters, vol. 89, no. 22, Article ID 221122, 2006. 
[3] A. Snigirev, V. Kohn, I. Snigireva, and B. Lengeler, "A compound refractive lens for focusing high-energy X-rays," Nature, vol. 384, no. 6604, pp. 49-51, 1996.

[4] V. Aristov, M. Grigoriev, S. Kuznetsov et al., "X-ray refractive planar lens with minimized absorption," Applied Physics Letters, vol. 77, no. 24, pp. 4058-4060, 2000.

[5] C. G. Schroer and B. Lengeler, "Focusing hard X rays to nanometer dimensions by adiabatically focusing lenses," Physical Review Letters, vol. 94, no. 5, Article ID 054802, 2005.

[6] K. Evans-Lutterodt, A. Stein, J. M. Ablett, N. Bozovic, A. Taylor, and D. M. Tennant, "Using compound kinoform hardX-ray lenses to exceed the critical angle limit," Physical Review Letters, vol. 99, no. 13, Article ID 134801, 2007.

[7] S.-L. Chang, YU. P. Stetsko, M.-T. Tang et al., "X-ray resonance in crystal cavities: realization of fabry-perot resonator for hard X rays," Physical Review Letters, vol. 94, no. 17, Article ID 174801, 2005.

[8] S.-L. Chang, Yu. P. Stetsko, M.-T. Tang, et al., "Fabry-Perot resonator for hard X rays: a diffraction experiment," Physical Review B, vol. 74, Article ID 134111, 7 pages, 2006.

[9] S.-Y. Chen, Y.-Y. Chang, M.-T. Tang et al., "Multi-plate crystal cavity with compound refractive lenses," in Advances in $X$ Ray/EUV Optics and Components III, vol. 7077 of Proceedings of SPIE, San Diego, Calif, USA, August 2008.

[10] Y.-Y. Chang, S.-Y. Chen, H.-H. Wu et al., "Diffractionenhanced beam-focusing for X-rays in curved multi-plate crystal cavity," Optics Express, vol. 18, no. 8, pp. 7886-7892, 2010.

[11] A. Caticha and S. Caticha-Ellis, "Dynamical theory of x-ray diffraction at Bragg angles near $\pi / 2$," Physical Review B, vol. 25, no. 2, pp. 971-983, 1982.

[12] V. G. Kohn, YU. V. Shvydko, and E. Gerdau, "On the theory of an X-ray Fabry-Perot interferometer," Physica Status Solidi B, vol. 221, no. 2, pp. 597-615, 2000.

[13] M. Yabashi, K. Tamasaku, S. Kikuta, and T. Ishikawa, "Xray monochromator with an energy resolution of $8 \times 10^{-9}$ at $14.41 \mathrm{keV}$," Review of Scientific Instruments, vol. 72, no. 11, pp. 4080-4083, 2001.

[14] A. Authier, Dynamical Theory of X-Ray Diffraction, Oxford University Press, Oxford, UK, 2001.

[15] S.-L. Chang, X-Ray Multiple-Wave Diffraction: Theory and Application, Spinger, Berlin, Germany, 2004.

[16] S. Kikuta, Y. Imai, T. Iizuka, Y. Yoda, X.-W. Zhang, and K. Hirano, "X-ray diffraction with a Bragg angle near $\pi / 2$ and its applications," Journal of Synchrotron Radiation, vol. 5, no. 3, pp. 670-672, 1998.

[17] J. P. Sutter, E. E. Alp, M. Y. Hu et al., "Multiple-beam xray diffraction near exact backscattering in silicon," Physical Review B, vol. 63, no. 9, Article ID 094111, 12 pages, 2001.

[18] M.-S. Chiu, YU. P. Stetsko, and S.-L. Chang, "Dynamical calculation for X-ray 24-beam diffraction in a two-plate crystal cavity of silicon," Acta Crystallographica Section A, vol. 64, no. 3, pp. 394-403, 2008. 